\title{
Frequency response as a surrogate eigenvalue problem in topology optimization
}

\author{
Andreassen, Erik; Ferrari, Federico; Sigmund, Ole; Diaz, Alejandro Rafael
}

Published in:

International Journal for Numerical Methods in Engineering

Link to article, DOI:

10.1002/nme.5563

Publication date:

2018

Document Version

Peer reviewed version

Link back to DTU Orbit

Citation (APA):

Andreassen, E., Ferrari, F., Sigmund, O., \& Diaz, A. R. (2018). Frequency response as a surrogate eigenvalue problem in topology optimization. International Journal for Numerical Methods in Engineering, 113(8), 12141229. https://doi.org/10.1002/nme.5563

\section{General rights}

Copyright and moral rights for the publications made accessible in the public portal are retained by the authors and/or other copyright owners and it is a condition of accessing publications that users recognise and abide by the legal requirements associated with these rights.

- Users may download and print one copy of any publication from the public portal for the purpose of private study or research.

- You may not further distribute the material or use it for any profit-making activity or commercial gain

- You may freely distribute the URL identifying the publication in the public portal 


\section{Frequency response as a surrogate eigenvalue problem in topology optimization}

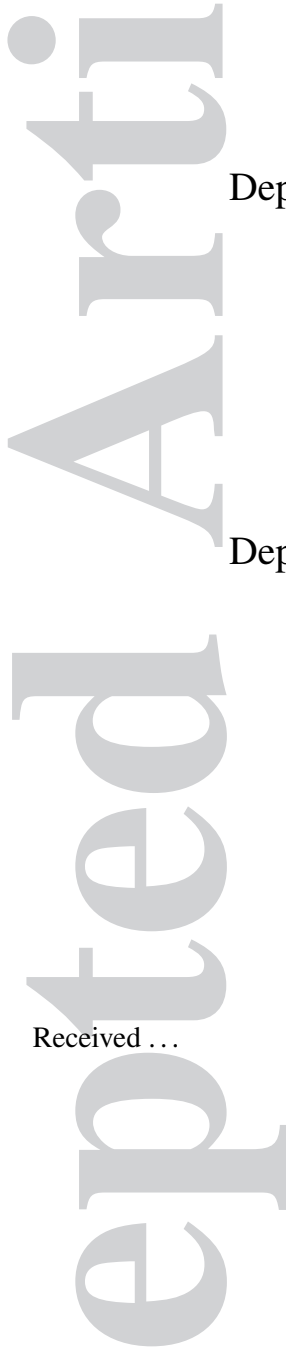

Erik Andreassen

Department of Mechanical Engineering, Solid Mechanics, DTU

email: erand@mek.dtu.dk

Federico Ferrari

Dipartimento di Ingegneria "Enzo Ferrari"

Università degli Studi di Modena e Reggio Emilia

email: federico.ferrari@unimore.it

Ole Sigmund

Department of Mechanical Engineering, Solid Mechanics, DTU

email: sigmund@mek.dtu.dk

Alejandro R. Diaz

Department of Mechanical Engineering, MSU

email: diaz@egr.msu.edu

Maximizing the fundamental eigenvalue is a basic goal in structural optimization. When self-weight is small compared to non-structural masses, optimized structures are very similar to compliance optimized structures. However, convergence to degenerated designs, as often seen for single load compliance problems, is prevented due to the load independent eigenvalue objective. For cases with small or no non-structural masses, optimized structures may be quite different from compliance designs. The first works on topology optimization with fundamental frequency objectives are found

This article has been accepted for publication and undergone full peer review but has not been through the copyediting, typesetting, pagination and proofreading process, which may lead to differences between this version and the Version of Record. Please cite this article as doi: $10.1002 / \mathrm{nme} .5563$ 
in $[10,33,20,19]$. In the case of repeated eigenvalues, simple eigenvalue gradients are no longer valid and must be treated as suggested in [26, 27, 25].

Whereas the fundamental eigenfrequency of a structure usually is of great importance, some situations may require different dynamic objectives such as maximum eigenvalue separation [17], dynamic response for a given frequency [24, 18, 32, 29], frequency response over a broader frequency range $[29,16,11]$, maximization of band gaps in periodic composites [29, 28, 29], or optimization of transient response [9].

In this paper we concentrate on the fundamental eigenvalue problem, however, we use an indirect technique to maximize it inspired by frequency response techniques, c.f. $[24,22,18,32,29,13$, $16,11]$. In those papers, frequency response as e.g. dynamic compliance is optimized for given excitation frequencies. Here we propose a dynamic update of this driving frequency which indirectly maximizes the fundamental eigenfrequency. We also refer to recent independent work by Olhoff and $\mathrm{Du}$ [23] who suggest an incremental frequency technique somewhat related to ours, however, requiring occasional solutions of eigenvalue problems that we try to avoid. The ideas and concepts developed here also apply to closely associated buckling problems which will be considered in future work.

In general, large scale topology optimization problems involving eigenvalues, such as those arising in dynamics and stability problems, present significant challenges. Special features of these problems that make their solution challenging include

1. Eigenvalue multiplicity

Repeated eigenvalues are a common occurrence in optimized designs and they often lead to loss of differentiability and poor convergence. Selecting which modes must be included in the optimization can be difficult.

2. A large number of eigenvalues and eigenvectors must be computed

As eigenvalues coalesce, many modes must be included in the analysis to account for possible mode switching, even if the goal is to maximize only the fundamental eigenvalue.

3. Computational complexity

Large scale eigenvalue problems can be difficult to solve.

Whereas the first issue appears to have been largely resolved, issues 2 and 3 represent presently unresolved, challenging obstacles. These obstacles prevent the development of reliable and efficient approaches for large scale structural optimization, e.g. involving millions of design variables $[1,8]$.

During optimization iterations (or from the very beginning in case of symmetric structures), the lower eigenvalues will start clustering and eventually coalesce, resulting in eigenvalue multiplicity. Multiple eigenvalue may slow down convergence of eigenvalue solvers and cause loss of differentiability. The latter problem can be solved (c.f. [26, 27]) but this still leaves arbitrary decisions regarding criteria for characterizing multiplicity and requires specialized optimization formulations that can handle varying number of constraints (c.f. active set strategies).

Even when one aims at maximizing the first eigenvalue and multiplicity is low, [7, 12] report that a very large number of eigenvalues and modes is required for robust convergence of the optimization algorithm, due to mode switching. For seemingly simple plate problems [7] needs to calculate the first 100 eigenvalues (the active set) and for a moderately sized 3D problem (144,000 design variables), [12] report that the first 50 eigenvalues and modes are required for stable convergence,

This article is protected by copyright. All rights reserved. 
even when optimizing for just the fundamental mode! These two references deal with buckling problems but similar conclusions can be drawn for dynamic problems.

The solution of large scale eigenvalue problems is still a standing challenge. The first reason is sheer computational resources. To date, the largest reported number of design variables for stiffnessbased topology optimization problem is 114 million [1], run on 1800 cores for 4.5 hours. Even if one had access to a direct solver, solving for 50 or more eigenvalues in a problem of this size would probably require orders of magnitude more computational effort. Furthermore, due to the complexity of the eigenvalue space and the large number of closely spaced eigenvalues, there is no guaranty that all eigenvalues will be found. To make matters worse, for really large problems, direct solvers have huge memory demands and do not scale well and one must resort to iterative schemes, applied on problems that are not positive definite and ill-conditioned due to large material contrast. Convergence will be very dependent on the availability of efficient preconditioning (multilevel, domain-decomposition, etc.) which at present is not well-developed for this purpose. Even with an effective iterative solver and preconditioner, the computational effort of solving such large scale eigenvalue problems is still prohibitively high due to the number of solutions of linear systems required by modern Arnoldi/Lanczos procedures and the need for solutions for several shift values.

Based on this discussion we conclude that the computational effort and other remaining challenges associated with the solution of large scale, multiple eigenvalue problems are hindering the development of topology optimization formulations that include stability constraints (buckling) or design consideration based on structural frequencies. We focus here on the latter. For very large problems, one should seek alternative formulations that achieve the goal of maximizing the fundamental frequency without requiring solution of the large scale, multiple eigenvalue problem.

In this paper we discuss the use of frequency response to eliminate the need for solving eigenvalue problems when maximizing the fundamental frequency in undamped, free vibration. By minimizing a measure of "dynamic compliance", we show that for excitation frequencies approaching the fundamental frequency, minimizing dynamic compliance corresponds to maximizing the fundamental frequency. The approach is demonstrated on a couple of $2 \mathrm{D}$ continuum test cases. It is motivated first by means of a simple three design variable problem, used to fix ideas and to expose some relevant features.

Although we here provide the original idea and demonstrate initial feasibility, many obstacles have to be overcome before the approach can be applied to practical, large scale topology optimization problems. Nevertheless, we are confident that these obstacles can be overcome, and then the computational effort of the approach will correspond to that of solving standard compliance problems for a limited number of simple linear load cases.

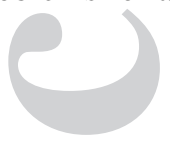

\section{STANDARD FORMULATIONS FOR EIGENVALUE TOPOLOGY OPTIMIZATION IN} DYNAMICS

To fix ideas we start from the statement of the underlying eigenvalue problem and its associated optimization. The standard eigenvalue problem in dynamics is

$$
(K-\Lambda M) \phi=0
$$

This article is protected by copyright. All rights reserved. 
where $\Lambda$ is the eigenvalue, $\phi$ is the eigenvector, $K$ is the stiffness and $M$ is the mass matrix. Solutions to this problem come in pairs $\left(\Lambda_{i}, \phi_{i}\right)$. We assume that both $K$ and $M$ are symmetric and positive definite. Then the eigenvalues are positive and real and can be ordered such that

$$
0<\Lambda_{1} \leq \Lambda_{2} \leq \Lambda_{3} \leq \cdots
$$

$\Lambda_{1}$ is the eigenvalue associated with the fundamental frequency $\Omega_{1}$, i.e., $\Lambda_{1}=\Omega_{1}{ }^{2}$. To simplify notation, in the following we will use $\Lambda$ and not $\Omega^{2}$ but we may refer to it as the frequency.

The underlying optimization problem involving the fundamental frequency as design criterion is of the form

$$
\max _{\boldsymbol{x} \in S} \Lambda_{1}, \quad \text { s.t. (1) }
$$

where $\boldsymbol{x}$ denotes the vector of design variables and $S$ represents the feasible set, e.g.,

$$
S=\left\{x: \sum x_{i} \leq V, \quad 0<x_{\min } \leq x_{i} \leq 1\right\}
$$

\section{AN ALTERNATIVE OPTIMIZATION FORMULATION BASED ON FREQUENCY RESPONSE}

Associated with (1) is the following linear frequency response problem:

$$
(\boldsymbol{K}-\lambda \boldsymbol{M}) \boldsymbol{w}=\boldsymbol{F}
$$

In this problem, the system is excited at a frequency $\lambda\left(=\omega^{2}\right)$ by an external force $\boldsymbol{F}$, resulting in a dynamic response of amplitude $\boldsymbol{w}$ at the same frequency. Matrix

$$
\boldsymbol{K}^{D}(\boldsymbol{x} ; \lambda)=\boldsymbol{K}(\boldsymbol{x})-\lambda \boldsymbol{M}(\boldsymbol{x})
$$

is the "dynamic stiffness" of the system excited at a frequency $\lambda$. In this scenario, the magnitude of the response can be measured by the dynamic compliance of the structure,

$$
C: C(\boldsymbol{x} ; \lambda)=\boldsymbol{w}^{T} \boldsymbol{K}^{D} \boldsymbol{w}
$$

When the excitation frequency $\lambda$ approaches $\Lambda_{1}$, the dynamic stiffness is reduced. As a result, for a general load $\boldsymbol{F}$, the amplitude $\boldsymbol{w}$ of the dynamic response increases, eventually becoming unbounded when $\lambda=\Lambda_{1}$. This suggests the following optimization problem, parameterized by $\lambda\left(\lambda<\Lambda_{1}\right)$ :

$$
\min _{\boldsymbol{x} \in S}|C(\boldsymbol{x} ; \lambda)|, \quad \text { s.t. }(3)
$$

This is the frequency response optimization problem. Here we investigate this problem as an alternative to the standard eigenvalue optimization formulation (2) that can produce maximum or near maximum eigenvalues without actually solving an eigenvalue problem at all.

This article is protected by copyright. All rights reserved. 


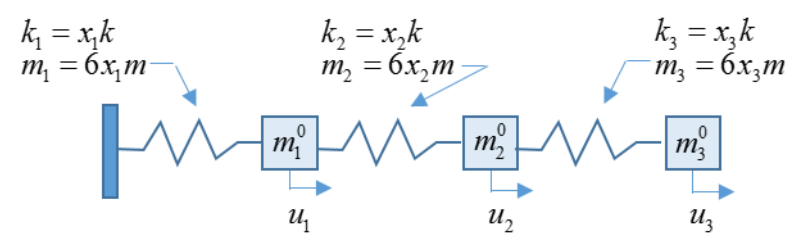

(a) Spring arrangement

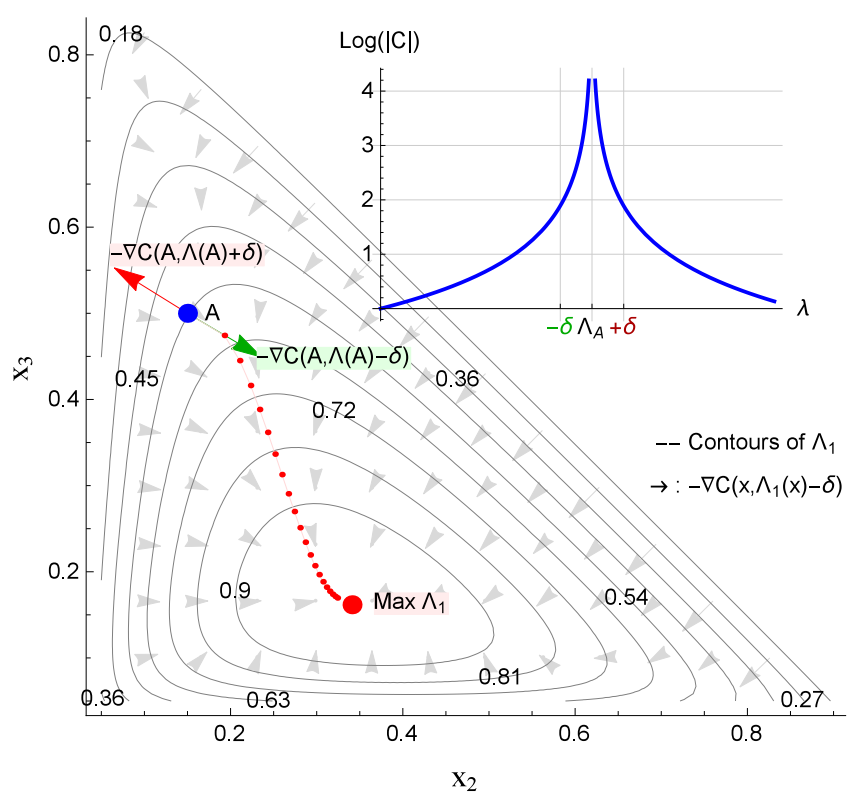

(b) Contours of $\Lambda_{1}$ and gradients of $C$

Figure 1. 3-dof example

\subsection{Motivation: A simple three-dof system}

To fix ideas and to expose the principal features of using frequency response as a surrogate for eigenvalue optimization, we look first into a simple, three-dof system, shown in Figure(1a). In this simple example three masses $m^{0}{ }_{1}=m, m^{0}{ }_{2}=m, m^{0}{ }_{3}=2 m$ are connected by three linear springs that also contribute to the total mass. Spring stiffness and masses are proportional to design parameters $x_{1}, x_{2}$, and $x_{3}$, respectively - one for each spring. The undamped, free vibration of this system is modeled by eigenvalue problem (1) with

$$
\boldsymbol{K}=k\left(\begin{array}{ccc}
x_{1}+x_{2} & -x_{2} & 0 \\
-x_{2} & x_{2}+x_{3} & -x_{3} \\
0 & -x_{3} & x_{3}
\end{array}\right)
$$

This article is protected by copyright. All rights reserved. 
We seek to maximize the first eigenvalue of this system by varying $x_{1}, x_{2}$, and $x_{3}$. A resource constraint and bounds on the parameters are added to define the feasible set. We use

$$
S=\left\{\left(x_{1}, x_{2}, x_{3}\right), x_{1}+x_{2}+x_{3} \leq 1,0<x_{i} \leq 1, i=1,2,3\right\}
$$

For non-zero design independent masses $m^{0}$ the resource constraint is active at the optimum solution. Thus, it is possible to reduce the problem to two design variables, $x_{2}$ and $x_{3}$, setting $x_{1}=1-x_{2}-x_{3}$. Figure (1b) shows the two-dimensional feasible set and the location of the optimum solution. Finally, the scale factor $k / m$ is adjusted so that the maximum eigenvalue $\Lambda_{1}$ in the feasible set is $\Lambda_{1}=1$.

Now consider a gradient-based approach to maximize the first mode eigenvalue, e.g., starting from point $A$ in Figure (1b). In this setting, one would construct a descent direction for $\Lambda_{1}$ at A and move in the opposite direction (to maximize $\Lambda_{1}$ ). The gradient $\nabla \Lambda_{1}(\mathrm{~A})$ is used to build this direction. As we want to avoid solving any eigenvalue problem, we will construct the descent direction from a properly crafted frequency response problem, and avoid solving the eigenvalue problem altogether, as discussed next.

As an alternative to solving the eigenvalue problem directly, consider instead the following approach: excite system A at a given driving frequency $\lambda$ and compute the resulting response $\boldsymbol{w}$ and the dynamic compliance $C=\boldsymbol{w}^{T} \boldsymbol{K}^{D} \boldsymbol{w}$ and its gradient at A. Figure (1b) shows the gradient of $C$ at $\mathrm{A}$ for excitations $\lambda$ on either side of the resonance $\Lambda_{1}(\mathrm{~A})$. The inset in the figure shows the (log) dynamic compliance at $\mathrm{A}, C(\mathrm{~A} ; \lambda)$, as a function of excitation $\lambda$. We note that

1. Excitations below the resonance result in a descent direction $\boldsymbol{d}=-\nabla C(\mathrm{~A} ; \lambda)$ (green vector) that points towards increasing $\Lambda_{1}$ while excitations $\lambda$ above the resonance result in a direction pointing towards decreasing $\Lambda_{1}$ (red vector).

2. Gradients $\nabla C(\mathrm{~A} ; \lambda)$ are discontinuous and undefined at $\lambda=\Lambda_{1}(\mathrm{~A})$.

3. As the excitation $\lambda$ approaches $\Lambda_{1}(\mathrm{~A})$ from below, $\boldsymbol{d}=-\nabla C(\mathrm{~A} ; \lambda)$ becomes normal to the contour at $\mathrm{A}$ and points towards increasing the eigenvalue.

For this excitation load and with the appropriate choice of driving frequency $\lambda$, the previous observations hold everywhere in the feasible domain. Gradients of $C$ can be used as descent directions for the fundamental frequency $\Lambda_{1}$ anywhere in the feasible set (except of course at the optimum solution). This is illustrated in Figure(1b), which shows contours of $\Lambda_{1}$ and vectors $\boldsymbol{d}=-\nabla C\left(\boldsymbol{x} ; \Lambda_{1}(\boldsymbol{x})-\delta\right)$ throughout the feasible domain.

Using these ideas we show in Figure (1b) an optimization iteration sequence $\boldsymbol{x}^{\nu}, \nu=1,2, \ldots$, starting from $\boldsymbol{x}^{1}=\mathrm{A}$. In each step, a search direction $\boldsymbol{d}^{\nu}$ is set to $-\nabla C\left(\boldsymbol{x}^{\nu} ; \lambda^{\nu}\right)$ and $\lambda^{\nu}$ is chosen as $0.95 * \Lambda_{1}\left(x^{\nu}\right)$ (this is for illustration only, in practice we cannot do this, since we want to avoid computing $\Lambda_{1}$ !). The last point in the iteration sequence corresponds to an eigenvalue $\Lambda_{1}=0.998$, essentially the same as the maximum eigenvalue in the feasible domain.

These observations show that, at least in this simple example, minimizing the dynamic compliance function $C$ can be used as a surrogate objective in the maximization of $\Lambda_{1}$, provided that the

This article is protected by copyright. All rights reserved. 
excitation frequency remains close to and below the resonance frequency. Ensuring that $\lambda$ remains below the resonance without actually computing $\Lambda_{1}$ is crucial to the success of this strategy.

\subsection{Sequence of optimization subproblems}

The simple three-spring example serves to expose interesting features of the problem. Most importantly, it shows that, while the frequency response optimization problem does serve as a surrogate for the eigenvalue optimization problem, there are important caveats. In particular, the driving frequency $\lambda$ cannot remain constant throughout the optimization. Instead, the maximum eigenvalue solution can be approached only through a sequence of frequency response optimization subproblems, parametrized by $\lambda$. Each subproblem in the sequence $\nu=1,2, \ldots$ is of this form:

Problem $\mathbf{P}\left(\lambda^{\nu}\right)$ : Starting from $\boldsymbol{x}_{0}{ }^{\nu}$, find $\boldsymbol{x} \in S$ that

$$
\begin{array}{ll}
\text { minimizes } & C\left(\boldsymbol{x} ; \lambda^{\nu}\right)=\boldsymbol{w}^{T} \boldsymbol{K}^{D}\left(\boldsymbol{x} ; \lambda^{\nu}\right) \boldsymbol{w} \\
\text { subject to } & \left|x_{i}-x_{0}{ }^{\nu}{ }_{i}\right| \leq \Delta, \quad i=1, \ldots, n
\end{array}
$$

where

$$
\left(\boldsymbol{K}-\lambda^{\nu} \boldsymbol{M}\right) \boldsymbol{w}=\boldsymbol{F},
$$

the feasible set $S$ is used to control the amount of resources, e.g.,

$$
S=\left\{\boldsymbol{x}: \sum x_{i} \leq V, \quad 0<x_{\min } \leq x_{i} \leq 1\right\},
$$

and $\Delta$ is used to control step size. The solution to $\mathrm{P}\left(\lambda^{\nu}\right)$ is used as $\boldsymbol{x}_{0}{ }^{\nu+1}$ to start the next subproblem, $\mathrm{P}\left(\lambda^{\nu+1}\right)$.

The sequence of problems $\mathrm{P}\left(\lambda^{\nu}\right)$ must be generated carefully so that descent directions for $C\left(\boldsymbol{x} ; \lambda^{\nu}\right)$ for each problem in the sequence points in the right direction, namely, towards increasing $\Lambda_{1}$. If done carefully, this guarantees that the initial design in each sequence remains in the basin of attraction of solutions with increasing $\Lambda_{1}$. This depends crucially on $\lambda^{\nu}$ and $\boldsymbol{F}$ and a strategy to choose these parameters is discussed next.

\subsection{Selecting the driving frequency}

We focus first on the selection of $\lambda^{\nu}$. In order to make sure that descent directions in $\mathrm{P}\left(\lambda^{\nu}\right)$ lead to increasing eigenvalues one has to guarantee that $\lambda^{\nu}$ is close to and below the first eigenvalue of the system. There are several ways to achieve this. For instance, one may use Padé approximations to estimate the first natural frequency $[4,15,16]$. Here we use the following result from linear algebra:

If $K$ and $M$ are positive definite matrices, the number of eigenvalues $\Lambda$ in (1) smaller than $\lambda$ equals the number of negative entries in the diagonal matrix $D$ in the LU decomposition of $K^{D}$ as $\boldsymbol{K}^{D}=\boldsymbol{L D U}$.

This article is protected by copyright. All rights reserved. 
Noting that the LU decomposition of $K^{D}$ is needed to solve (8), learning weather a particular driving frequency $\lambda$ is below $\Lambda_{1}(x)$ requires little additional computational effort, especially if using a direct solver. However, to ensure that the same $\lambda$ remains close to and below $\Lambda_{1}(\boldsymbol{x})$ as $\boldsymbol{x}$ changes may require the solution of this simple (but potentially costly) one-dimensional, line-search problem that bounds the location of $\Lambda_{1}(\boldsymbol{x})$ :

Problem L $\left(\lambda^{\max } ; \boldsymbol{x}\right)$ : Let $n(\lambda)$ be the number of negative entries in $\boldsymbol{D}$ in the LU decomposition of $\boldsymbol{K}^{D}(\boldsymbol{x} ; \lambda)$. Starting from $\lambda=\lambda^{\max }>0$,

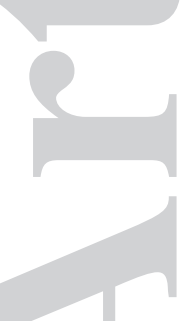

$$
\begin{aligned}
\operatorname{maximize} & \lambda \\
\text { subject to } & n(\lambda)=0 \\
& 0<\lambda \leq \lambda^{\max }
\end{aligned}
$$

Under the stated assumptions, Problem $\mathrm{L}\left(\lambda^{\max } ; \boldsymbol{x}\right)$ has a solution for any positive $\lambda^{\max }$. The problem can be solved using a simple line-search algorithm, for instance, one that brackets the solution in intervals $\left[\lambda_{\text {low }}, \lambda_{\text {hi }}\right]$ of decreasing size that contain $\Lambda_{1}(\boldsymbol{x})$. We note that

1. Any feasible solution to $\mathrm{L}\left(\lambda^{\max } ; \boldsymbol{x}\right)$ is a lower bound for $\Lambda_{1}(\boldsymbol{x})$.

2. $\Lambda_{1}(\boldsymbol{x}) \in\left[\lambda_{\text {low }}, \lambda_{\text {hi }}\right]$ if $n\left(\lambda_{\text {low }}\right)=0$ and $n\left(\lambda_{\text {hi }}\right)=1$.

3. To obtain a good estimate of $\Lambda_{1}(\boldsymbol{x}), \lambda^{\text {max }}$ should be fixed to a value larger than the expected maximum eigenvalue solution.

4. Larger values of $\lambda^{\max }$ and tighter intervals $\left[\lambda_{\text {low }}, \lambda_{\mathrm{hi}}\right]$ lead to more computational expense, as each line-search iteration requires a decomposition of $K^{D}$. They also result in better estimates of $\Lambda_{1}(x)$.

Problem $\mathrm{L}\left(\lambda^{\max } ; \boldsymbol{x}_{0}{ }^{\nu}\right)$ can be used to set the driving frequency $\lambda^{\nu}$. For instance, if after a couple of iterations the bracket $\left[\lambda_{\text {low }}, \lambda_{\text {hi }}\right]$ contains $\Lambda_{1}(\boldsymbol{x})$, setting $\lambda^{\nu}$ to a value slightly lower than $\lambda_{\text {low }}$ leads to robust iteration strategies. We use this strategy in the examples discussed below, in Section 4.

\subsection{Selecting the driving force}

Finally, the driving force $\boldsymbol{F}$ in (8) needs to be defined. Implicit in the discussion above is the assumption that $\boldsymbol{F}$ can excite the vibration mode associated with $\Lambda_{1}(\boldsymbol{x})$ and that this will hold for any design $x$. As we will expose in the examples, prescribing such excitation must be done carefully, particularly in problems where repeated eigenvalues may occur. It is easy to see that the response $\boldsymbol{w}$ is of the form

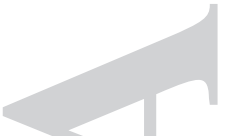

$$
\boldsymbol{w}=\sum_{i} \frac{f_{i}}{\lambda-\Lambda_{i}} \boldsymbol{\phi}_{i}
$$

where $\phi_{i}$ is the $i$ - th eigenmode (see (1) ) and $f_{i}$ is the projection of $\boldsymbol{F}$ onto the mode $\boldsymbol{\phi}_{i}$, that is, the dot-product (modal load factor) $f_{i}=\boldsymbol{\phi}_{i}{ }^{T} \boldsymbol{F}$. . Evidently, the response from a load $\boldsymbol{F}$ with $f_{1}=0$ will not exhibit a resonance behavior about $\Lambda_{1}$. To illustrate, consider again the simple three-spring example from Section 3.1. Two responses corresponding to two different excitation forces $\boldsymbol{F}^{(1)}$

This article is protected by copyright. All rights reserved. 


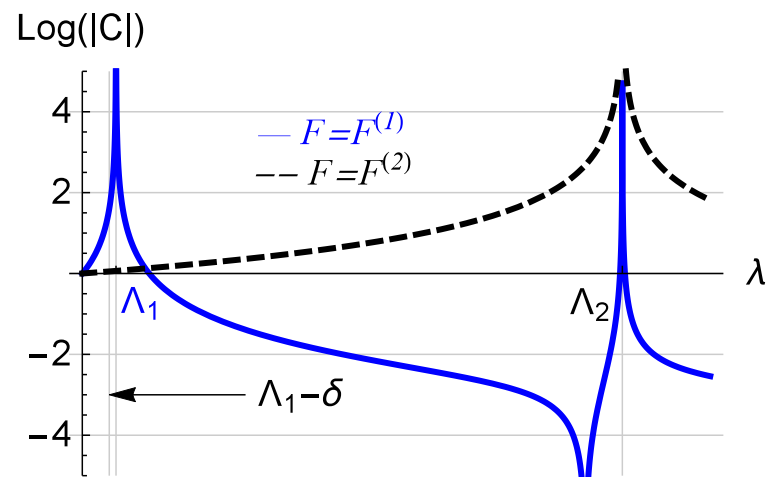

Figure 2. Simple three-spring example: frequency response from two excitations. $\boldsymbol{F}^{(2)}$ does not excite the first mode

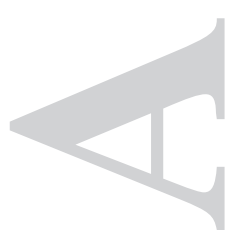

and $\boldsymbol{F}^{(2)}$ are shown in Figure 2. $\boldsymbol{F}^{(1)}$ excites both modes but $\boldsymbol{F}^{(2)}$ is chosen deliberately to be orthogonal to $\phi_{1}$ and it is obvious that the corresponding response shows no resonance near $\Lambda_{1}$. In computations, if $\boldsymbol{F}^{(2)}$ was the only load applied, the excitation frequency $\lambda$, selected by the line search algorithm (10), would be slightly to the left of $\Lambda_{1}(x)$, far from the resonance, resulting in an essentially static response.

The simple example illustrates the importance of selecting an excitation load that excites the first mode. In problems where there is potential for eigenvalues coalescing near $\Lambda_{1}$, it is important that the excitation force excites all modes corresponding to the coalescing eigenvalues. As the number of possible coalescing eigenvalues is not known a priori, a good approach is to consider multiple load cases from the start, including a rich enough set of loads in the optimization problem. This suggest the following multiple-load alternative to (7):

Problem $\mathbf{P}\left(\lambda^{\nu}\right)$ (multiple loads): Starting from $x_{0}{ }^{\nu}$, find $\boldsymbol{x} \in S$ that

$$
\begin{array}{ll}
\text { minimizes } & \hat{C}\left(\boldsymbol{x} ; \lambda^{\nu}\right)=\sum_{l} \boldsymbol{w}^{(l)^{T}} \boldsymbol{K}^{D}\left(\boldsymbol{x} ; \lambda^{\nu}\right) \boldsymbol{w}^{(l)} \\
\text { subject to } & \left|x_{i}-x_{0}{ }^{\nu}{ }_{i}\right| \leq \Delta, \quad i=1, \ldots, n
\end{array}
$$

where

$$
\left(\boldsymbol{K}-\lambda^{\nu} \boldsymbol{M}\right) \boldsymbol{w}^{(l)}=\boldsymbol{F}^{(l)},
$$

and $l$ indexes different load cases $\boldsymbol{F}^{(l)}, l=1,2, \ldots N_{l}$

We note that the sensitivities of $\boldsymbol{F}^{(l)}$ are not included in the sensitivity analysis. There are many possible choices of loading vectors which will result in similar results as long as the appropriate eigenmodes are excited, however, if the vectors are included in the sensitivity analysis the optimizer may exploit this, in turn causing unwanted results.

This article is protected by copyright. All rights reserved. 
3.5. The overall strategy

The overall strategy has been tested using the method of moving asymptotes (MMA) algorithm [30] to solve the subproblems $\mathrm{P}\left(\lambda^{\nu}\right)$ in a standard density-based topology optimization setting (c.f. [3]), starting from the uniform density design, through the following steps:

- Step 1: Set $\nu=1$ and choose the initial design $x_{0}{ }^{\nu}$. For instance use the uniform density design to start the process.

- Step 2: Choose a driving frequency $\lambda^{\nu}$ such that $\lambda^{\nu}<\Lambda_{1}\left(x_{0}{ }^{\nu}\right)$. Here we use the line search problem $\mathrm{L}\left(\lambda^{\max } ; \boldsymbol{x}_{0}{ }^{\nu}\right)$ to compute a lower bound $\lambda_{\text {low }}$ for $\Lambda_{1}\left(\boldsymbol{x}_{0}{ }^{\nu}\right)$ and set

$$
\lambda^{\nu}=\alpha \lambda_{\text {low }}
$$

for a (prescribed) value of $\alpha$ close to (but below) 1 .

- Step 3: Solve $P\left(\lambda^{\nu}\right)$ for $\boldsymbol{x}^{*}$ starting from $\boldsymbol{x}_{0}{ }^{\nu}$.

- Step 4: If convergence is detected, $\boldsymbol{x}^{*}$ is the solution. Otherwise, set $\boldsymbol{x}_{0}{ }^{\nu+1}=\boldsymbol{x}^{*}, \nu=\nu+1$ and return to step 2 .

In practice,

- $\lambda^{\max }$ in step 2 is set sufficiently large so that it is larger than any expected value of $\Lambda_{1}$ in the feasible set.

- Line search iterations continue until a feasible solution is found and the final interval $\left[\lambda_{\text {low }}, \lambda_{\text {hi }}\right]$ is small, e.g., less than $5 \%$ of $\lambda_{\text {low }}$.

- $\lambda^{\nu}$ is typically set to 95 to $99 \%$ of $\lambda_{\text {low }}$.

- Only one iteration of the MMA algorithm is applied at step 3. In other words, the MMA is used only to compute a feasible direction and step size starting from $x_{0}{ }^{\nu}$.

- $\lambda^{\nu}$ can be used as an estimator of $\Lambda_{1}\left(\boldsymbol{x}_{0}{ }^{\nu}\right)$.

- A simple criterion for convergence is used: a combination of small step $\left|x_{0}{ }^{\nu+1}-x_{0}{ }^{\nu}\right|$ or slow improvement $\left|\lambda^{\nu+1}-\lambda^{\nu}\right|$.

We should emphasize here that our goal is simply to explore the potential of alternative methods to solve eigenvalue optimization problems, and we made no special effort to streamline computations. Thus, while the performance of this algorithm is not bad, it can certainly be improved, for instance, by focusing on better line search strategies $L$ to estimate $\lambda^{\nu}$ and more efficient ways to improve the solution within each subproblem P.

\section{EXAMPLES OF TOPOLOGY OPTIMIZATION}

The performance of the approach is explored using three simple examples, presented next. In all cases, a SIMP model $[5,21,35]$ is used to interpolate material properties, elastic modulus $E$ and material density $\rho$, via

$$
\begin{array}{r}
E(x)=E_{L}+x^{p}\left(E_{H}-E_{L}\right) \\
\rho(x)=\rho_{L}+x\left(\rho_{H}-\rho_{L}\right)
\end{array}
$$

This article is protected by copyright. All rights reserved. 


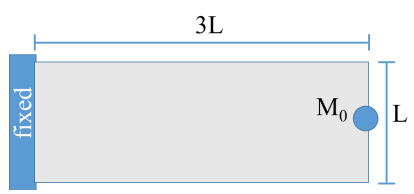

Figure 3. Example 1: Cantilever beam, geometry

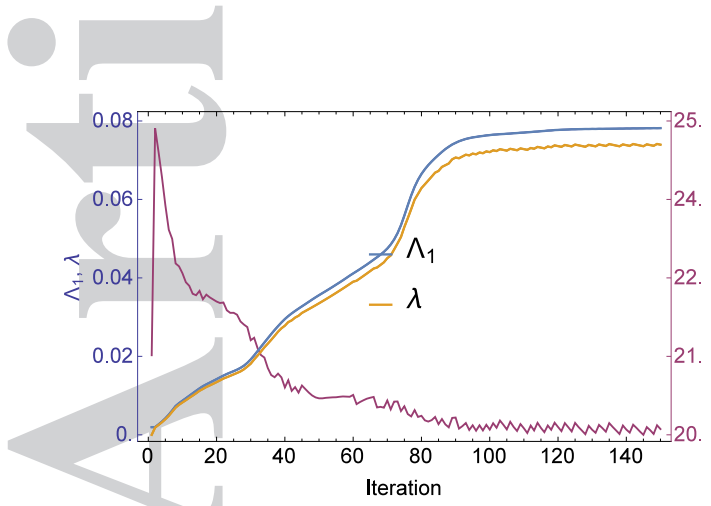

(a) Iteration history, $\lambda^{\nu}=0.95 \lambda_{\text {low }}$

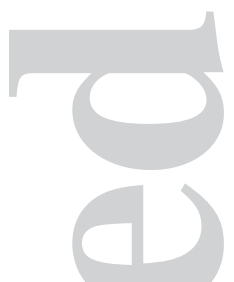

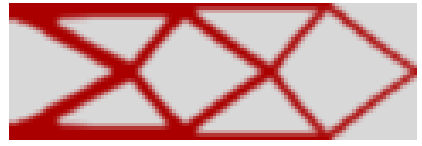

(c) Final layout from $\min C$

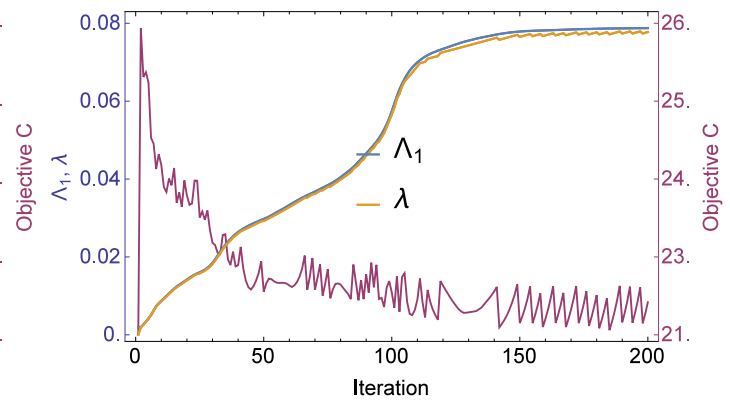

(b) Iteration history, $\lambda^{\nu}=0.99 \lambda_{\text {low }}$

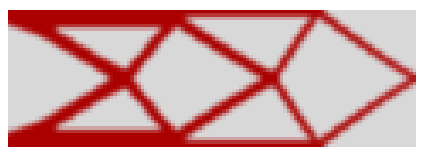

(d) Final layout from max $\Lambda_{1}$

Figure 4. Example 1: Cantilever beam, results

where $\left[E_{L}, E_{H}\right]=\left[10^{-3}, 1\right],\left[\rho_{L}, \rho_{H}\right]=\left[10^{-3}, 1\right]$ and $p=4$. Poisson's ratio $\nu=0.3$ is kept constant. A projection filter ensures checkerboard-free and mesh-independent solutions using a projection filter with radius $r=2$ (unless otherwise noted), threshold value $\eta=1 / 2$ and a small projection factor of $\beta=1$ [34]. The finite element model is standard: all elements are square, 4node elements in 2D elasticity. The total amount of material is constrained by $V=v \times N_{e}$ in (9), where $v$ is the volume fraction of solid material and $N_{e}$ is the total number of elements. In the optimization subproblems $\mathrm{P}\left(\lambda^{\nu}\right)$, the step size $\Delta$ is set to 0.05 . The process is started from the uniform density design, and the first iteration uses static compliance as the objective function (i.e., driving frequency $\lambda$ is set to 0 ).

\subsection{Cantilever Beam}

This example is based on the classical cantilever beam topology optimization problem. The example is used to look into the convergence of the algorithm and its ability to reproduce the solution of the maximum eigenvalue problem, exploring the effect of certain parameters on convergence.

The setup is standard: the design domain is rectangular with $L=1 / 3$, all displacements at the left wall are constrained (see Figure 3). A non-structural mass $M_{0}$ is attached mid-height on the right end with magnitude set to $20 \%$ of the allowed mass. $120 \times 40$ square elements are used. The volume fraction is $v=0.3$.

Figure 4 summarizes the results. The driving frequency $\lambda^{\nu}$ is selected using the line search algorithm L (10). Figures $4 \mathrm{a}$ and $4 \mathrm{~b}$ show iteration histories for different values of $\alpha$ in (14), 
the parameter that controls the gap between the driving frequency $\lambda^{\nu}$ and the eigenvalue $\Lambda_{1}$. As expected, for $\alpha$ close to $1, \lambda^{\nu}$ is a good estimate of the true eigenvalue $\Lambda_{1}$, also displayed in the figures (of course, $\Lambda_{1}$ is not used in the algorithm, it is shown only for the purpose of comparison). Choosing $\lambda^{\nu}$ closer to $\Lambda_{1}$, as in $4 \mathrm{~b}$, results in slightly better answers but also leads to slower convergence and more erratic $C$ iteration history (the jagged shape of the iteration curve $C$ is caused by the adjustment made to the driving frequency between iterations of the MMA algorithm). However, even when the iteration history for $C$ is not smooth, the corresponding sequence of $\Lambda_{1}$ is smooth and $\Lambda_{1}$ increases essentially monotonically, confirming the effectiveness of the approach. In this case, both sequences converge to essentially the same layout, shown in $4 \mathrm{c}$. For comparison, the layout obtained from direct maximization of $\Lambda_{1}$ is shown in Figure 4c. Direct maximization of $\Lambda_{1}$ yields $8.0256 \times 10^{-2}$ while minimizing $C$ yields slightly lower values: $\Lambda_{1}=7.8179 \times 10^{-2}$ when $\lambda^{\nu}=0.95 \lambda_{\text {low }}$ (Figure 4a) and $\Lambda_{1}=7.8758 \times 10^{-2}$ when $\lambda^{\nu}=0.99 \lambda_{\text {low }}$ (Figure 4b).

For this example the external load $\boldsymbol{F}$ was set as

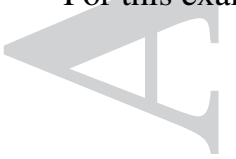

$$
F_{i}=c_{0} \sum_{j} M_{i, j}
$$

where $c_{0}$ is a positive but otherwise arbitrary scaling factor. Indices $i$ and $j$ represent degrees-offreedom, $M_{i, j}$ is the $i, j$ entry in the (structural) mass matrix $M$, and the same load is applied to both nodal degrees-of-freedom (horizontal and vertical). Only one load case was considered.

Choosing $\boldsymbol{F}$ as the sum of mass terms in (15) sets the force to be a distributed load proportional to the "lumped mass" assigned to each node. Applying a distributed load is beneficial in that all regions of the structure will be loaded, thus increasing the chances that several modes be excited, as opposed to, e.g., a point load, which could be more easily orthogonal to one or more of the relevant modes. Making the load proportional to the mass matrix scales the force in such a way that the force is automatically reduced in regions of the design space not occupied by material. The choice of loading is less crucial in this example, as the eigenvalues remain well separated throughout the optimization process and it is relatively easy to excite the first mode. The example in the following section shows a different scenario, one where coalescence of eigenvalues can lead to sub-optimal solutions if the load is not chosen carefully.

We note here that, as long as the force $F$ excites the relevant modes, its magnitude and spatial distribution should not determine the outcome. As we know, in a standard frequency response problem in vibrations, the structure will be excited at resonance by a whole range of forces. The location of the resonances in the frequency spectrum is independent of the loads (again, as long as the load can excite the relevant mode). For this reason, we repeat that we do not consider $\boldsymbol{F}$ in the computation of sensitivity information, arguing that, as in the standard frequency response problem in vibrations, the location and mode of the resonance are independent of the load.

We finish this example with a modification to the feasible set, adding a constraint on the (static) mean compliance. With this modification, the feasible set $S$ in (9) becomes

$$
S=\left\{\boldsymbol{x}: C_{0} \leq C_{\max }, \quad \sum x_{i} \leq V, \quad 0<x_{\min } \leq x_{i} \leq 1\right\},
$$

where $C_{0}=\boldsymbol{u}^{T} \boldsymbol{F}^{0}$ is the compliance resulting from the application of a static load $\boldsymbol{F}^{0}$ and the solution to the system

This article is protected by copyright. All rights reserved. 


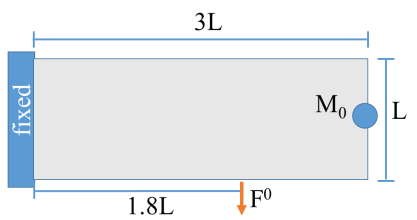

(a) Example 1: Cantilever beam geometry with static load
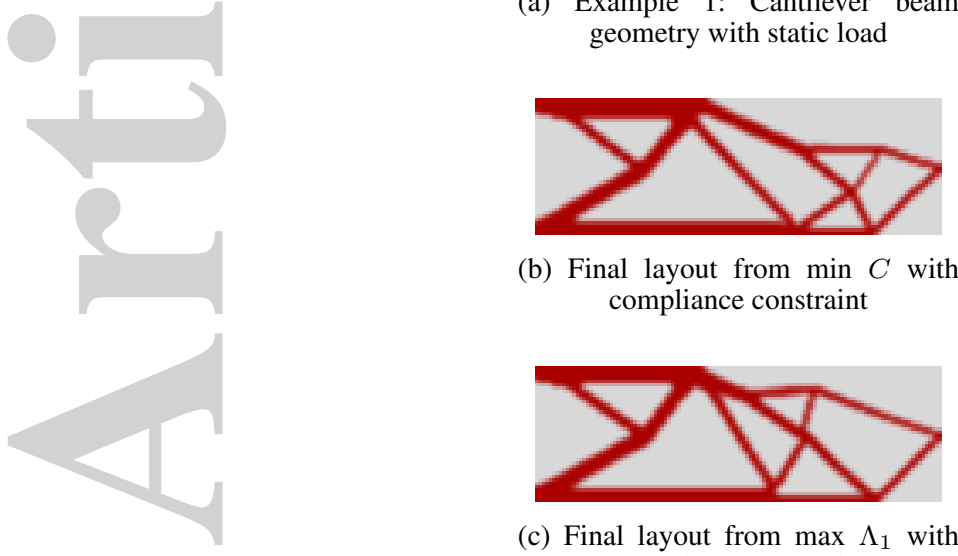

(b) Final layout from $\min C$ with compliance constraint

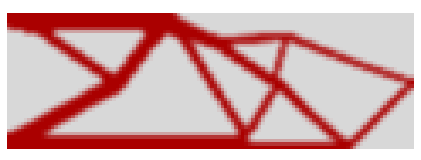

(c) Final layout from max $\Lambda_{1}$ with compliance constraint

Figure 5. Example 1: Cantilever beam, results with static compliance constraint

$$
K u=F^{0}
$$

A downward unit load $F^{0}$ is applied as shown in Figure 5a and $C_{\max }$ is set to half the static compliance associated with the first solution (Figure $4 a$ ). Here we show in Figure $5 b$ the results obtained using the surrogate frequency response problem P (7) and, for comparison, in Figure $5 \mathrm{c}$ the result obtained using the standard eigenvalue maximization problem. The final layouts are slightly different - this is not surprising, as these problems are not convex and potentially have many local minima, reachable from different paths in the feasible set generated by the solution algorithm. However, the performance associated with both layouts is rather similar, respectively $\Lambda_{1}=7.3670 \times 10^{-2}$ and $\Lambda_{1}=7.2624 \times 10^{-2}$, the surrogate problem yielding the slightly better answer!

\subsection{Cross - repeated eigenvalues}

For this example, the geometry is as shown in Figure (6). The problem is set up so that repeated eigenvalues are likely at the optimum solution. Boundary conditions are as shown in the figure and the design domain is almost square: $L_{x}=1$ and $L_{y}=0.9$. Three nodes are fully restrained at the center of each outer edge. A non-structural mass $M_{0}$ is placed at the center of the domain with magnitude set to $20 \%$ of the mass of the structure. $80 \times 72$ square elements are used. The volume fraction is $v=0.2$. The design (but not the displacement field) is fixed to be horizontally and vertically symmetric, i.e. only a quarter of the elements are used as design variables. In this way we force the optimization to stay in the challenging multiple eigenvalue situation.

This article is protected by copyright. All rights reserved. 
The choice of load is crucial in this problem. As in the previous example, we apply massproportional loads, but in this case the proportionality constant $c: c(x, y)$ is spatially varying, allowing excitation of symmetric and anti-symmetric modes independently, i.e.,

$$
F_{i}=c\left(x_{i}, y_{i}\right) \sum_{j} M_{i, j}
$$

where $\left(x_{i}, y_{i}\right)$ is the location of the node associated with degree-of-freedom $i$. The results shown below were obtained using sinusoidal variations of the form

$$
c^{(m, n)}\left(x_{i}, y_{i}\right)=c_{0} \sin \left(\frac{m \pi x_{i}}{L_{x}}\right) \sin \left(\frac{n \pi y_{i}}{L_{y}}\right), \quad m, n=1,2, \ldots
$$

We consider first only one load case, corresponding to $(m, n)=(1,1)$. This load is asymmetric (it excites the structure in a 45 degree direction). The results are summarized in Figure 7. Figure 7 a shows the iteration history. In this problem the first two eigenvalues are almost repeated at the initial design. As iterations progress, they grow while remaining close, until they cross with the third eigenvalue at iteration 46 (Figure 7b). After this point, the two originally lowest eigenvalues continue growing, whereas the originally third eigenvalue stagnates. The reason is that the original third mode, which becomes the lowest mode after 46 iterations, is a rotational mode (oscillating around the center), which is not excited by the applied single load. Hence, it does not contribute to $C$ and hence is not further improved. This aspect is further illustrated in $7 \mathrm{c}$, which shows that the modal load factor $f_{3}$ is zero throughout the iteration history and hence this mode is never excited. In contrast, original modes 1 and 2 have high modal load factors $f_{1}$ and $f_{2}$ throughout. It is hence clear that the single load case surrogate problem does not account well for this case of multiple active eigenvalues. The final layout is shown in Figure 7d, with corresponding $\Lambda_{1}=1.2093$. Clearly this design is not very efficient towards rotational vibrations unlike the optimized structure coming up next.

We now consider four separate load cases, $\boldsymbol{F}^{(1)}$ through $\boldsymbol{F}^{(4)}$, corresponding to $(m, n)=$ $(1,1),(1,2),(2,1)$ and $(2,2)$ in (19). These loads will excite symmetric as well as anti-symmetric and rotational modes. The results are summarized in Figure 8. Considering the iteration history in Figure $8 \mathrm{~b}$, as iterations progress, the first two modes move together throughout iterations, while this time eigenmodes 3 (and 4) are also excited and hence increase as well. This aspect is further illustrated in Figure 8c which shows the sum of modal load factors for each mode. The combination of all loads thus excites the first four modes throughout the iteration history and, as a result, they all contribute to the objective function $\hat{C}$, resulting in a successful outcome of the optimization process. The final layout is shown in Figure 8d, with $\Lambda_{1}=2.002$, much higher than before. It is seen how the optimized structure now also is stiff with respect to rotational vibration and hence the third eigenmode has this time indeed contributed to the optimization process.

\subsection{Computational efficiency}

The previous examples have demonstrated feasibility of the proposed approach. However, rather costly line search operations were used in order to update the frequency at each step. Nevertheless, numerical studies indicate that the CPU time, even for the small examples considered, is comparable to solving the standard eigenvalue optimization problem. This last example suggests a simplified

This article is protected by copyright. All rights reserved. 


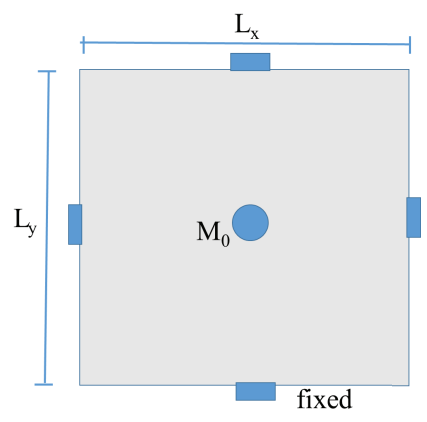

Figure 6. Example 2: Cross, geometry

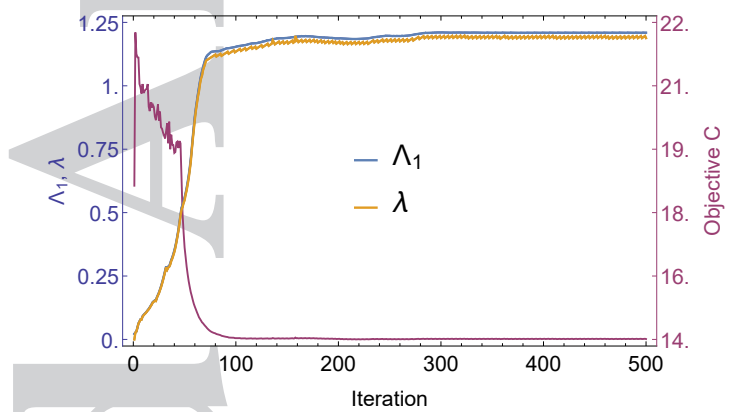

(a) Iteration history

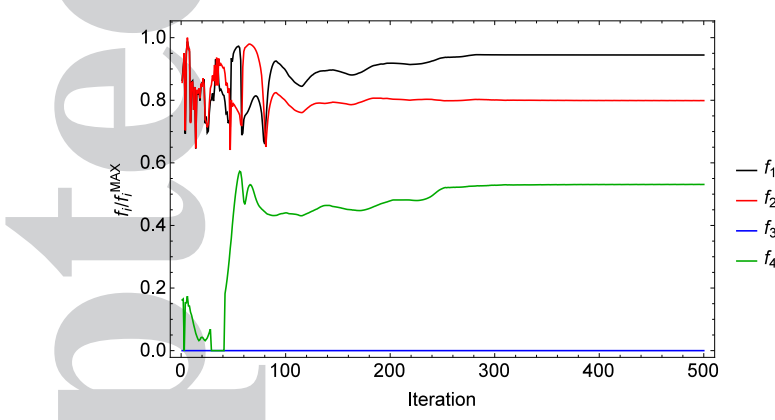

(c) Sum of modal load factors $f_{i}$ for each mode

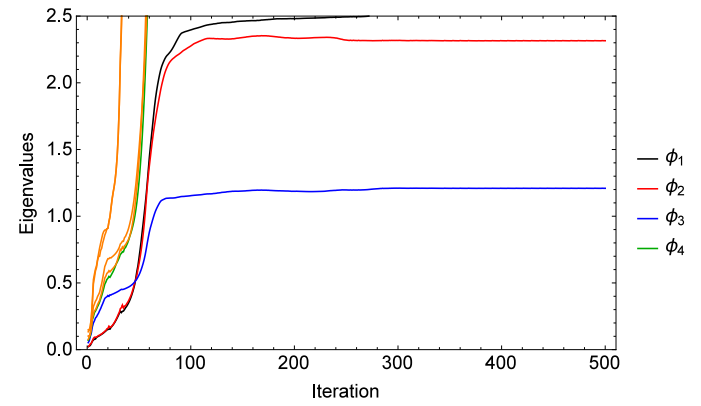

(b) Eigenvalues colored according to mode shape

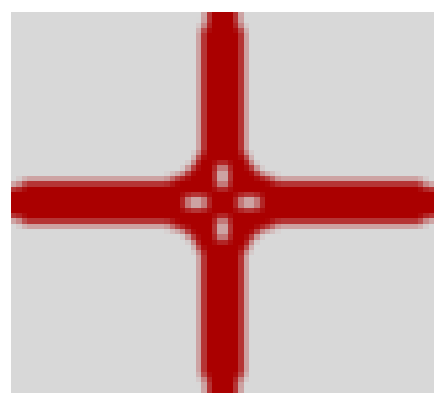

(d) Final layout

Figure 7. Example 2: Cross, results using one load case

procedure that even for small scale problems shows large speed improvements compared to the standard eigenvalue formulation. We envision many possibilities for further savings but deeper studies on efficiency improvements are left for future work.

We solve again the cantilever beam example of Sec. 4.1 now for four mesh resolutions $\mathcal{M}_{1}=120 \times 40, \mathcal{M}_{2}=240 \times 80, \mathcal{M}_{3}=480 \times 160$ and $\mathcal{M}_{4}=720 \times 240$. The driving frequency is updated once every 25 iterations, performing 10 steps of the bracketing algorithm discussed in Sec. 3.3. The standard eigenvalue optimization problem involves solving for the three lowest eigenvalues for each optimization step.

The final topologies, shown in Figure 9, are nearly indistinguishable from the corresponding ones obtained with standard eigenvalue optimization. However, Figure 9a shows that due to the occasional update of $\lambda$, the evolution of $\Lambda_{1}$ using the surrogate approach is quite different from the direct approach. Nonetheless, the increase is essentially monotonic and the final values of $\Lambda_{1}$ are

This article is protected by copyright. All rights reserved. 


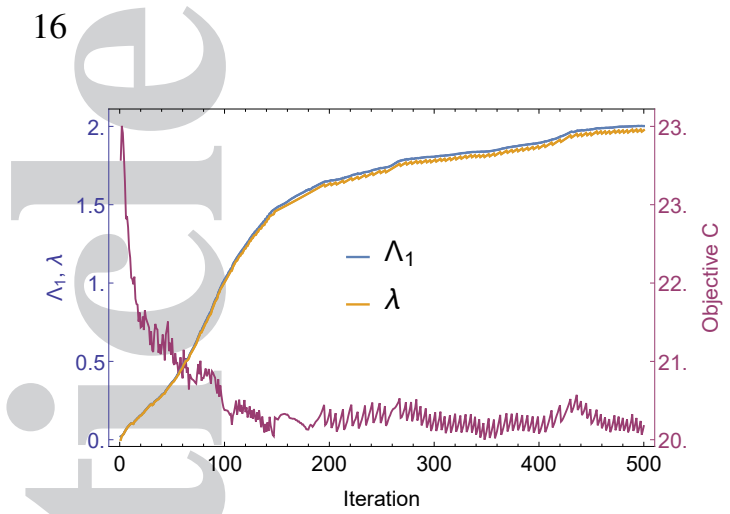

(a) Iteration history

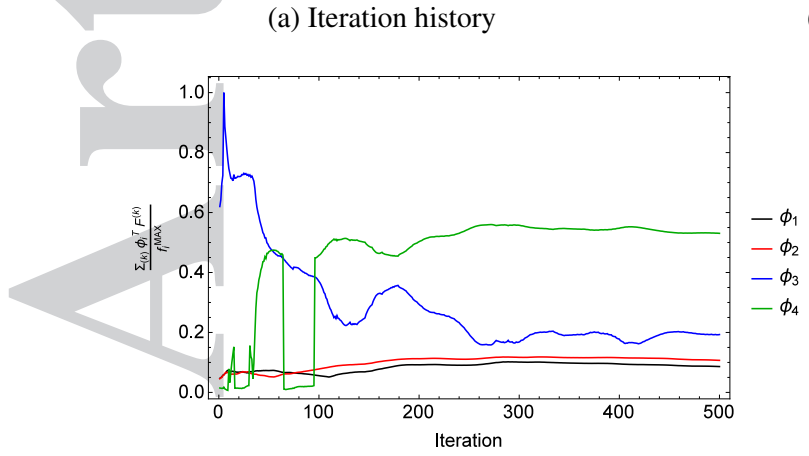

(c) Combined modal load factors $f_{i}$ as sum of modal load factors from each load case

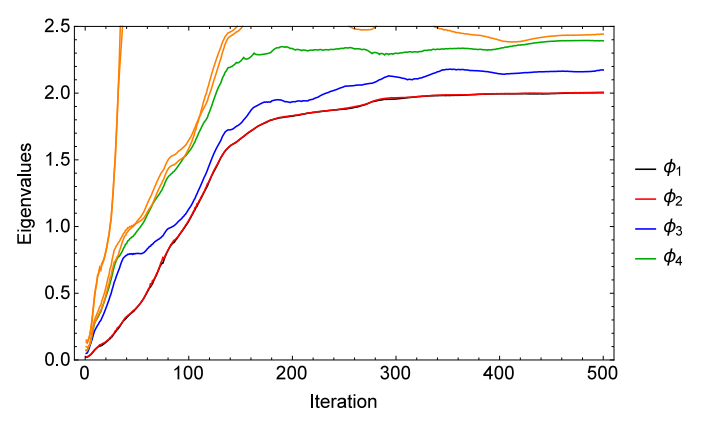

(b) Eigenvalues colored according to mode shape

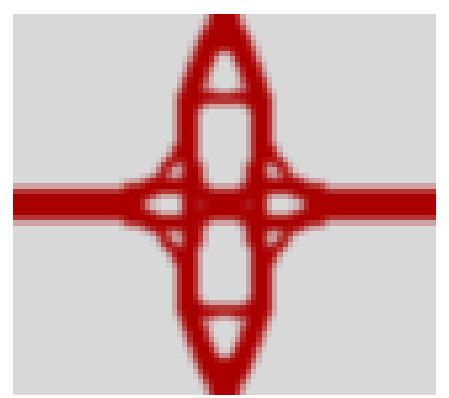

(d) Final layout

Figure 8. Example 2: Cross, results using four load cases

very close. Interestingly, the surrogate approach seems to converge somewhat slower for the finer mesh resolutions. We attribute this to the changing loads of the surrogate problem. Essentially, every 25 iterations the load case of the associated dynamic compliance problem is changed and hence the topology will have to adapt to this. For finer meshes, adaption to varying loading conditions is slower.

In each case the final value of the fundamental frequency obtained with the surrogate approach is just a few percent lower than the one obtained from the direct approach, see Tab. I. CPU times (obtained for a single processor Matlab implementation) are listed in Tab.I and scaling curves are shown in Figure 10. Comparing data and figures it is clear that quite significant savings can be achieved with the surrogate approach even for the small problems considered in this example. The predominant cost of the procedure is due to the solution of the linear system and not to the search for the driving frequency. The more than five times reduction in CPU time comes at the cost of a slight reduction in the achieved eigenfrequencies and somewhat increased iteration numbers.

Above timing study is based on standard Matlab implementations. That is, all system matrices are stored in sparse format and the standard backslash operator is used for linear system solving and subroutine "eigs" is used for solving for the five lowest eigenvalues in the standard formulation. Further savings may be achievable by exploitation of solver options or alternative solvers.

This article is protected by copyright. All rights reserved. 
Final $\Lambda_{1}\left(10^{-2}\right) \quad$ Computational time (s)

\begin{tabular}{ccccccccc}
\hline$n_{\text {dof }}$ & standard & surrogate & r.d. & $T_{\lambda}$ & $T_{C}$ & $t_{l s}$ & $t_{f u}$ & $T_{C} / T_{\lambda}$ \\
\hline 9,840 & 8.0422 & 7.9902 & 0.6 & 39.6 & 6.4 & 4.7 & 1.7 & 0.161 \\
38,880 & 7.6113 & 7.5697 & 0.6 & 168.7 & 29.2 & 21.1 & 8.1 & 0.173 \\
154,560 & 7.5654 & 7.3750 & 2.5 & 740.6 & 143.9 & 104.0 & 39.9 & 0.194 \\
347,040 & 7.5543 & 7.3590 & 2.6 & 1895.8 & 359.3 & 257.4 & 101.9 & 0.189 \\
\hline
\end{tabular}

Table I. Performance of the standard and surrogate formulations for four discretizations. The first two columns list the final value of the eigenvalue and the third contains relative differences. The next columns report the computational time for the operations described in Figure 10. The last column shows the time savings achieved with the new surrogate approach.

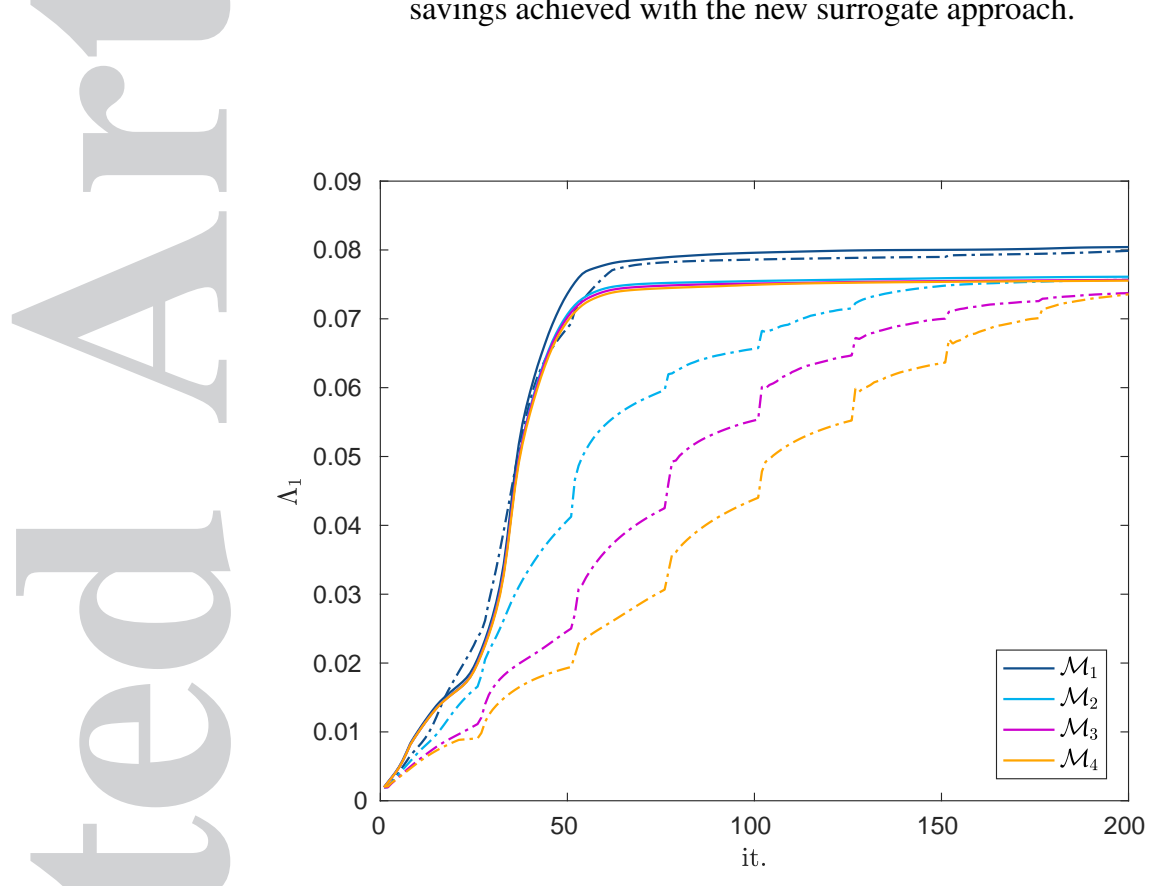

(a) Evolution of the fundamental eigenvalue from direct eigenvalue optimization (solid curves) and from frequency response minimization with occasional frequency update (dashed curves)

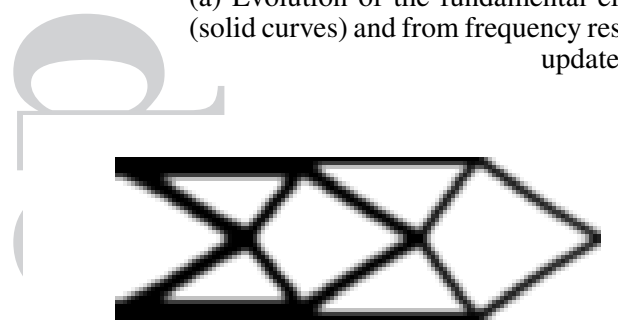

(b) $\mathcal{M}_{1}$

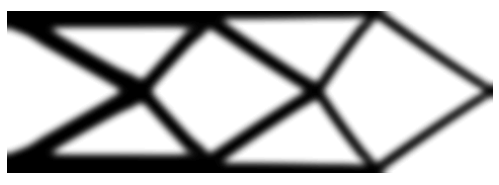

(d) $\mathcal{M}_{3}$

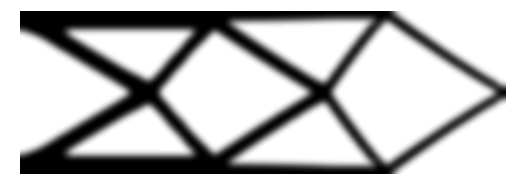

(c) $\mathcal{M}_{2}$

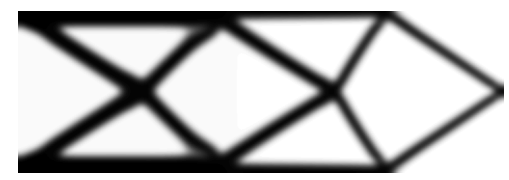

(e) $\mathcal{M}_{4}$

Figure 9. Comparison of eigenvalue evolution curves and final topologies obtained with the frequency response surrogate formulation on the four meshes.

This article is protected by copyright. All rights reserved. 


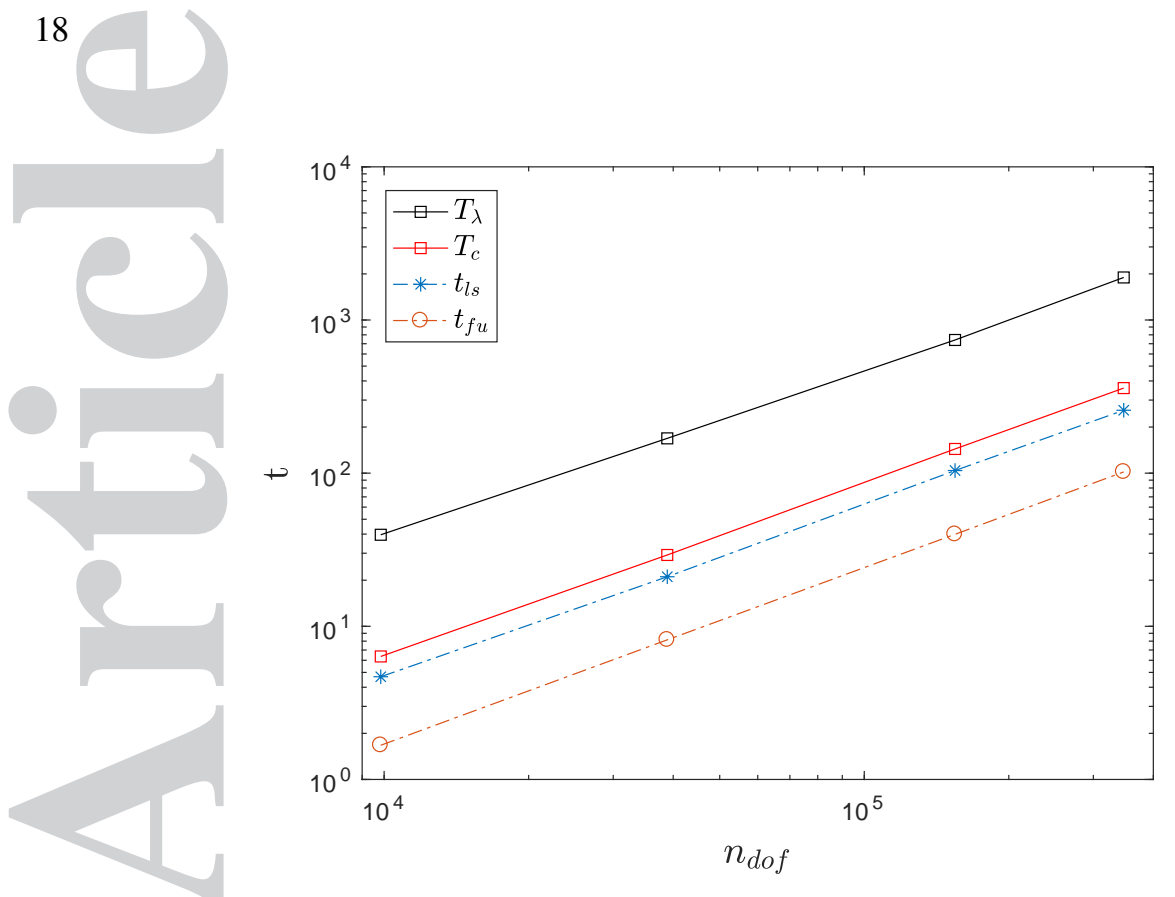

Figure 10. Scaling curves for the two approaches. $T_{\lambda}$ is the time required by eigenvalue analysis in the standard approach and $T_{c}$ is the time required by solving the state problem in the surrogate approach. The latter is split into the time for the solution of the linear system $\left(t_{l s}\right)$ and the time spent for searching the

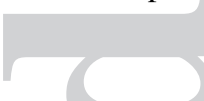
frequency $\left(t_{f u}\right)$

\section{CONCLUSIONS}

Adaptive frequency response optimization, as proposed, offers an alternative to solving the eigenvalue maximization problem without having to actually compute eigenvalues. The main application of this alternative approach is expected to be large scale problems and problems with multiple eigenvalues, where there may be no choice but to find an alternative strategy. However, the surrogate problem must be built carefully, choosing the proper driving frequency and a sufficiently complete set of loads, particulary when repeated eigenvalues are involved. Mass-proportional, spatially distributed loading appears to be an effective choice for external loading.

Computational efficiency was only explored briefly. The more time consuming and delicate part of the algorithm involves the adjustment of the driving frequency to be as close as possible but below the target eigenvalue. The initially formulated line search algorithm does the job, but at considerable expense. A simplified version with only occasional updates and reduced search points at potential saving factors in CPU time of more than five. Alternative approaches, e.g., using Padé approximations to estimate the frequency response curve, may potentially be even more efficient. While such investigations remain to be done, we believe that the evidence presented supports the use of frequency response as a surrogate for eigenvalue optimization and motivates further exploration of this approach.

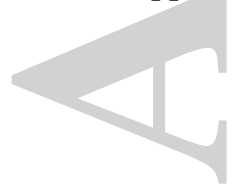

\section{ACKNOWLEDGEMENTS}

The first and third authors are sponsored by the Villum Fuundation through the NextTop project. Part of this work was performed while the fourth author was a visiting professor at the Technical

This article is protected by copyright. All rights reserved. 
University of Denmark, sponsored by the Mønsted Foundation. The second author wishes to thank Prof. Massimo Mancuso for fruitful discussions on the topic of the paper.

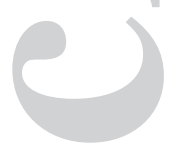

\section{REFERENCES}

1. N. Aage, E. Andreassen, and B.S. Lazarov. Topology optimization using PETSc: An easy-to-use, fully parallel, open source topology optimization framework. Structural and Multidisciplinary Optimization, 51(3):565-572, 2015.

2. S. Adali, M. Walker, and V.E. Verijenko. Multiobjective optimization of laminated plates for maximum prebuckling, buckling and postbuckling strength using continuous and discrete ply angles. Composite Structures, 35(1):117 - 130, 1996.

3. E. Andreassen, A. Clausen, M. Schevenels, B. Lazarov, and O. Sigmund. Efficient topology optimization in matlab using 88 lines of code. Structural and Multidisciplinary Optimization, 43:1-16, 2011. MATLAB code available online at: www.topopt.dtu.dk.

4. G.A. Baker and P.R. Graves-Morris. Padé approximants, volume 59. Cambridge University Press, 1996.

5. M.P. Bendsøe. Optimal shape design as a material distribution problem. Structural Optimization. 1:193-202, 1989.

6. M. P. Bendsøe and O. Sigmund. Topology Optimization - Theory, Methods and Applications. Springer Verlag, Berlin Heidelberg, 2004.

7. M. Bruyneel, B. Colson, and A. Remouchamps. Discussion on some convergence problems in buckling optimisation. Structural and Multidisciplinary Optimization, 35(2):181-186, 2008.

8. T.W. Chin and G. Kennedy. Large-scale compliance-minimization and buckling topology optimization of the undeformed common research model wing. In 57th AIAA/ASCE/AHS/ASC Structures, Structural Dynamics, and Materials Conference, page 0939, 2016.

9. J. Dahl, J. S. Jensen, and O. Sigmund. Topology optimization for transient problems in one dimension: Design of filters and pulse modulators. Structural and Multidisciplinary Optimization, 36:585-595, 2008.

10. A. R. Díaz and N. Kikuchi. Solution to shape and topology eigenvalue optimization problems using a homogenization method. International Journal for Numerical Methods in Engineering, 35:1487-1502, 1992.

11. M.B. Dühring, J.S. Jensen, and O. Sigmund. Acoustic design by topology optimization. Journal of Sound and Vibration, 317:557-575, 2008.

12. P.D. Dunning, E. Ovtchinnikov, J. Scott, and H.A. Kim. Level-set topology optimization with many linear buckling constraints using an efficient and robust eigensolver. International Journal for Numerical Methods in Engineering, 2016.

13. Du J, Olhoff N. Minimization of sound radiation from vibrating bi-material structures using topology optimization. Structural and Multidisciplinary Optimization 2007; 33(4-5):305-321.

14. A.R. de Faria. Prebuckling enhancement of beams and plates under uncertain loadings and arbitrary initial imperfections. Journal of the Brazilian Society of Mechanical Sciences and Engineering, 29(4):388-395, 2007.

15. J. S. Jensen and O. Sigmund. Topology optimization of photonic crystal structures: A high-bandwidth low-loss T-junction waveguide. Journal of the Optical Society of America B: Optical Physics, 22(6):1191-1198, 2005.

16. J.S. Jensen. Topology optimization of dynamics problems with Padé approximants. International journal for numerical methods in engineering, 72(13):1605-1630, 2007.

17. J.S. Jensen and N.L. Pedersen. On maximal eigenfrequency separation in two-material structures: the $1 \mathrm{~d}$ and $2 \mathrm{~d}$ scalar cases. Journal of Sound and Vibration, 289(4-5):967-986, 2006.

18. C. S. Jog. Topology design of structures subjected to periodic loading. J. of Sound and Vibration, 253:687-709, 2002.

19. T.S. Kim, J.E. Kim, and Y.Y. Kim. Parallelized structural topology optimization for eigenvalue problems. International Journal of Solids and Structures, 41(9-10):2623 - 2641, 2004.

20. Z.-D. Ma, N. Kikuchi, and H.-C. Cheng. Topological design for vibrating structures. Computer Methods in Applied Mechanics and Engineering, 121(1-4):259-280, 1995.

21. H.P Mlejnek. Some aspects of the genesis of structures. Structural Optimization, 5:64-69, 1992.

22. Nishiwaki S, Saitou K, Min SJ, Kikuchi N. Topological design considering flexibility under periodic loads. Structural and Multidisciplinary Optimization, 19:4-16, 2000.

23. N. Olhoff and J. Du. Generalized incremental frequency method for topological design of continuum structures for minimum dynamic compliance subject to forced vibration at a prescribed low or high value of the excitation frequency. Structural and Multidisciplinary Optimization, 54:1113-1141, 2016.

24. J. S. Ou and N. Kikuchi. Optimal design of controlled structures. Structural Optimization, 11:19-28, 1996.

This article is protected by copyright. All rights reserved. 
25. N. L. Pedersen. Maximization of eigenvalues using topology optimization. Structural and Multidisciplinary Optimization, 20(1):2-11, 2000.

26. A. P. Seyranian. Sensitivity analysis of multiple eigenvalues. Mechanics of Structures and Machines, 21:261-284, 1993.

27. A. P. Seyranian, E. Lund, and N. Olhoff. Multiple eigenvalues in structural optimization problems. Structural Optimization, 8(4):207-227, 1994.

28. O. Sigmund and K. Hougaard. Geometrical properties of optimal photonic crystals. Physical Review Letters, 100(15):153904, April 182008.

29. O. Sigmund and J. S. Jensen. Systematic design of phononic band gap materials and structures by topology

optimization. Philosophical Transactions of the Royal Society A: Mathematical, Physical and Engineering Sciences, 361:1001-1019, 2003.

30. K. Svanberg. A class of globally convergent optimization methods based on conservative convex separable approximations. SIAM Journal of Optimization, 12:555-573, 2002.

31. F. Wang, B.S. Lazarov, O. Sigmund, and J.S. Jensen. Interpolation scheme for fictitious domain techniques and topology optimization of finite strain elastic problems. Computer Methods in Applied Mechanics and Engineering, 276:453-472, 2014.

32. D. Tcherniak. Topology optimization of resonating structures using SIMP method. International Journal for Numerical Methods in Engineering, 54(11):1605-1622, 2002.

33. L. H. Tenek and I. Hagiwara. Static and vibrational shape and topology optimization using homogenization and mathematical programming. Computer Methods in Applied Mechanics and Engineering, 109(1-2):143-154, 1993.

34. F. Wang, B.S. Lazarov, and O. Sigmund. On projection methods, convergence and robust formulations in topology optimization. Structural and Multidisciplinary Optimization, 43(6):767-784, 2011.

35. M. Zhou and G.I.N. Rozvany. The COC algorithm, part II: Topological, geometry and generalized shape optimization. Computer Methods in Applied Mechanics and Engineering, 89:197-224, 1991.

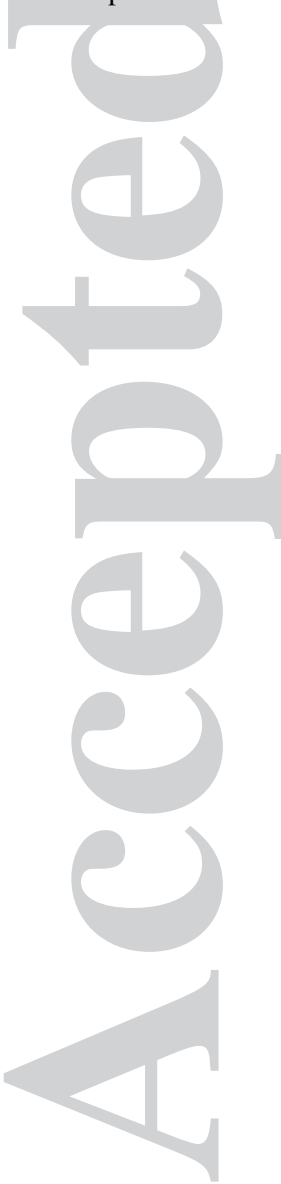

This article is protected by copyright. All rights reserved. 